Mots. Les langages du politique

Présidentielle 2007. Scènes de genre

\title{
Un espace public local au miroir d'un journal de quartier
}

Bruno Raoul

\section{OpenEdition}

Journals

Édition électronique

URL : https://journals.openedition.org/mots/19087

DOI : $10.4000 /$ mots. 19087

ISSN : 1960-6001

Éditeur

ENS Éditions

Édition imprimée

Date de publication : 1 juillet 2009

ISBN : 978-2-84788-151-6

ISSN : 0243-6450

\section{Référence électronique}

Bruno Raoul, « Un espace public local au miroir d'un journal de quartier », Mots. Les langages du politique [En ligne], 90 | 2009, mis en ligne le 30 mars 2011, consulté le 23 avril 2022. URL : http:// journals.openedition.org/mots/19087 ; DOI : https://doi.org/10.4000/mots.19087

\section{(c) ENS Éditions}




\section{Un espace public local au miroir d'un journal de quartier}

Dans le cadre d'une recherche en cours portant sur des médias associatifs de quartier diffusés dans une grande ville du Nord de la France, nous étudions ces médias dans leur fonction d'expression au sein de l'espace public local et sous l'angle de leur contribution à la production du territoire dudit quartier. Jusqu'ici, nous nous sommes particulièrement intéressé à Quartier libre, publié par un comité de quartier depuis 1980. Ce journal en était, en février 2008, à son $176^{\text {e numéro }}{ }^{1}$. Il est distribué dans toutes les boites aux lettres d'un quartier d'habitat ancien dont la configuration et le bâti témoignent de l'histoire industrielle de la ville. Peuplé d'environ 12000 habitants, celuici a été répertorié par l'INSEE, sur la base du recensement de 1999, comme quartier de "pauvreté extrême " $^{2}$. La requalification du bâti et des espaces publics et la redynamisation de l'activité menées sous l'égide de la municipalité depuis une dizaine d'années dans le cadre des programmes nationaux de rénovation urbaine ont conduit à redonner de l'attractivité à ce quartier. Il n'en reste pas moins dans une situation fragile, ce dont témoigne également, comme on le verra, le taux d'abstention aux élections.

Nous nous proposons, dans cet article, d'approcher ce journal dans sa fonction de médiation, et cela à partir de l'analyse de sa production discursive. Considérer cette fonction de médiation, c'est certes envisager le média en ce qu'il contribue à rendre visibles, voire à soumettre à la discussion critique, dans un espace public donné, tant des questions du quotidien que des questions de société. Nous cherchons donc à comprendre en quoi un média de quartier participe de la mise en forme et de la mise en sens du débat public dans un cadre spatial délimité, en quoi il contribue à façonner un espace

Université Lille 3, Gériico, bruno.raoul@univ-lille3.fr

1. Son rythme de publication s'est modifié au cours du temps: de 3 à 4 numéros par an lors de sa première décennie d'existence, il est passé à un tirage mensuel dans les années quatre-vingt-dix pour se stabiliser plus récemment à une moyenne bimestrielle. Il est tiré à 5000 exemplaires, la plupart du temps sous le format de quatre pages A4. Sa maquette a également connu quelques évolutions. Depuis la fin de 2005, il est imprimé en quadrichromie sur papier glacé et réserve une place plus importante qu'auparavant à la photographie.

2. INSEE, 2007. 
public local au point de rencontre de l'attachement au quartier et de l'engagement politique (Neveu, 2007, p. 37). Mais derrière l'idée de médiation, dont on considère ici la «matérialité discursive» qui lui donne "forme publique» (Delforce, Noyer, 1999, p. 36), il s'agit aussi de cerner ce que le texte du journal peut nous révéler de ce qui semble faire (ou ne plus faire) sens pour ses producteurs 3 , de pointer quelques-uns des ressorts sociologiques et politiques qui fondent le journal et expliquent son évolution. Outre le fait d'appréhender un aspect de ce qui fait «le lien inscrit dans le lieu» (Boure, Lefebvre, 2000, p. 271), nous proposons un regard sur les dynamiques sociales d'un quartier inscrites sous l'enseigne de la participation (en prenant toutefois appui sur une approche différente de celle d'autres travaux concernant ce thème: Authier et al., 2007; Neveu, 2003; Blondiaux, 2000; Lefebvre, Nonjon, 2003).

Sur le plan méthodologique, nous intéressant au discours d'un type de médias spécifique (propre à un territoire bien délimité, émanant d'un acteur de ce territoire dont la mission première n'est pas de produire un journal), il s'est agi moins de "construire » un corpus (d'articles) sur la base d'hypothèses qui le fondent a priori (Bonnafous, 2006; Krieg-Planque, 2006) que de réunir un corpus (de journaux) qui existe à l'état latent. Un tel « corpus clos» nous fait accéder à un genre de discours prédécoupé institutionnellement (Maingueneau, 2005, p. 72). Nous nous sommes attaché à interpréter, dans une perspective interdisciplinaire, la matière discursive du journal en portant plus spécialement notre attention, lors d'un premier repérage, sur les thèmes traités, le lexique, les formules discursives et les modalités énonciatives, sans oublier la forme même du média. Nous avons pu ainsi identifier des constantes, d'une part, des inflexions, voire des ruptures, d'autre part, et une investigation plus systématique nous a ensuite amené à travailler sur des points particuliers pour explorer plus profondément l'évolution de la publication.

Notre analyse privilégiant une perspective diachronique, nous avons relevé une évolution des valeurs qui sont au fondement de la publication: celle-ci est passée du régime discursif correspondant à une posture militante (structuration, argumentation dans la réflexion et les revendications) à un régime narratif (lequel n'exclut pas la contestation) concernant les réunions et autres évènements du quartier. Le discours verse de plus en plus dans l'anecdotique, voire, parfois, dans le registre de la dénonciation publique et dans une exploitation individualisante, quelque peu instrumentale, de la «parole habitante».

Dans cet article, en nous centrant sur trois thèmes d'investigation (l'interpellation du lecteur, l'interpellation de l'électeur, la parole des habitants) et donc en considérant trois types différents de rapport au public, nous repé-

3. Le journal est rédigé par la permanente et des membres du conseil d'administration de l'association. Il est régulièrement rappelé que ces derniers sont « des personnes bénévoles, habitant ou travaillant dans le quartier, n'ayant pas de mandat politique et désireuses de s'investir dans la vie du quartier». 
rerons les mouvements de la fonction de médiation du journal. Nous verrons aussi comment se décline, sur une dizaine d'années, un décalage progressif entre l'ambition originelle du comité de quartier et la difficulté que celui-ci rencontre aujourd'hui à faire montre, par ce moyen, d'une vigilance critique et argumentée dans l'espace public local, d'une capacité d'analyse et d'une force de proposition programmatique équivalentes à celles dont il a pu témoigner dans la première moitié des années quatre-vingt-dix 4 .

\section{De l'interpellation des lecteurs à la représentation des habitants : une médiation ambigüe}

Dans un petit encadré publié de manière récurrente de 1990 à 1996 sous l'intitulé «mode d'emploi», le comité de quartier se présente comme un «interlocuteur privilégié de la municipalité » et explique que son «but» est d'«organiser un débat ouvert entre les habitants sur les différents projets que peuvent avoir le maire et son équipe municipale». Cette formule subit toutefois une légère modification, début 1993, pour attribuer au comité un rôle en même temps moins circonscrit et plus vague: «[...] un débat ouvert entre les habitants sur le quartier dans tous les domaines». Pourtant, le journal laisse aussi transparaitre un autre objectif du comité: « Nous voulons mobiliser plus d'habitants pour faire changer le cadre de vie de ce quartier» ( ${ }^{\circ} 54$, janvier 1992). Il est précisé par la suite que « faire [les] réunions une fois par mois en invitant de temps en temps un élu est nécessaire mais pas suffisant pour obtenir les transformations que nous souhaitons pour le quartier» ( ${ }^{\circ} 55$, février 1992). II y a là, de manière sous-jacente, une autre dimension de l'enjeu de la démocratie participative (même si le terme n'est pas alors employé), et le journal en est le vecteur. En effet, sa fonction ne se limite pas à informer ni à engager des débats dans l'espace public: il est aussi un instrument d'interpellation des habitants dans une perspective de mobilisation militante. Et le style peut être parfois à l'injonction, typographie à l'appui - gros titre de une, caractères gras: «AVEC LE COMITÉ DE QUARTIER, PRENEZ VOTRE QUARTIER EN MAIN » ( ${ }^{\circ} 62$, novembre 1992) -, voire friser la provocation: « Ne restez pas passifs, prenez en mains votre vie au quotidien et votre avenir à votre porte; n'attendez pas qu'il soit trop tard, réagissez dès maintenant pour prévoir et préparer votre vie de demain et celle de nos enfants pour de longues années » (supplément du n 82, septembre 1994). À l'occasion, un dessin pourra être convoqué: "Le quartier a besoin de vous! » dit, en une, le personnage de l'oncle Sam, le plan (bien agrandi) du quartier dans une main, l'index de l'autre main pointé avec autorité vers le lecteur ( $\mathrm{n}^{\circ} 108$, janvier-février 1997).

4. Sur ce point, voir Raoul, 2006. 
Dans un style moins autoritaire, mais néanmoins pressant, on trouve: «Ne subissez pas sans réagir, ne laissez pas toujours les autres décider pour vous. Consacrez-nous quelques heures par mois. Rejoignez-nous au Comité de quartier. Faites-nous parvenir vos constats, vos remarques, vos propositions... » ( $n^{\circ} 163$, janvier 2006). Enfin, l’invitation peut être plus feutrée : «Pour qu'on ne relègue pas les Comités de quartier au Musée, section “Traditions du Passé” ", écrit ainsi un administrateur à l'occasion de la nouvelle année, «je vous souhaite pour l'année 2002 une profonde envie de changer votre vie» ( $n^{\circ} 138$, janvier 2002).

Dans son souci de se distinguer des institutions de la « démocratie représentative», le comité de quartier explique ainsi le sens de l'engagement de ses militants: "Car n'oublions surtout pas que, contrairement aux "vrais" élus issus des urnes de la République, qui n'arrivent à obtenir, à l'issue de deux tours de scrutin, qu'un pitoyable quart des suffrages exprimés, les bénévoles associatifs, acteurs dans la vie de leur quartier, ne perçoivent aucune indemnité. Alors qu'ils se dépensent tout autant sinon parfois plus pour l'intérêt public que ne le font les élus indemnisés... " ( $n^{\circ} 138$, janvier 2002, dans un article appelant à s'investir dans le conseil d'administration). À l'automne 2002, dans un éditorial au titre simplificateur et démagogique - «Tout se détache ou tous des taches» [sic] -, le président du moment invite les lecteurs, dans un style lyrique (il est question des «liens» et des «ponts de communication» entre les personnes et les territoires), à «reprendre [leur] place dans la gestion de la ville» et à «veiller à ne pas passer à côté de la chance qui [leur] est donnée de sauver la démocratie et les droits de tout un chacun». Le texte est dans la même veine que l'éditorial du numéro précédent, qui évoquait les «politiciens de complaisance» ( $n^{\circ} 144$, octobre 2002)5.

De tels appels aux lecteurs et, au-delà, aux habitants du quartier sont légitimés par la posture que revendique le comité de quartier : celle d'être non seulement le porte-parole de ces derniers, mais aussi le garant de leurs intérêts. De ce fait, Quartier libre apparait parfois comme le support sinon l'aiguillon de la contestation quand l'heure est à celle-ci (contre la municipalité, notamment), voire comme le moyen de lui donner forme et de la faire exister. En ce qui concerne les habitants eux-mêmes, ils sont envisagés comme pouvant et devant se mobiliser, notamment pour se faire entendre auprès de la municipalité et ne pas se laisser dessaisir du pouvoir qu'ils détiendraient collectivement. C'est la figure d'un habitant passif, d'un habitant « qui subit sans réagir »

5. À la suite de tels éditoriaux, le président du comité sera amené à démissionner. S'abritant derrière une formule convenue - «Pour des raisons professionnelles et personnelles, le Président a souhaité se libérer de sa fonction » ( $n^{\circ} 147$, mai-juin 2003) -, le journal se garde de revenir sur les raisons de cette démission. Mais bien que les numéros ultérieurs témoignent d'une recherche d'apaisement, ils font apparaitre la nécessité, pour la suite de notre recherche, d'explorer davantage les rapports du journal et de la municipalité. 
qui est ainsi dénoncée et qui fait pendant à celle d'un habitant-citoyen, acteur impliqué. Mais la récurrence de tels appels à l'implication peut aussi s'interpréter comme un indice de la difficulté du comité de quartier à mobiliser et donc à représenter les habitants. Finalement, en considérant l'ambigüité de l'expression «représentation des habitants» telle qu'elle peut s'entendre ici ("parler au nom des habitants», d'une part, «perception des habitants» par les responsables de l'association, d'autre part), on entrevoit en quoi la fonction de médiation du journal trahit un entre-deux révélateur de la difficulté du comité de quartier à afficher un positionnement clairement établi.

Un tel comité n'a de représentativité que celle qu'il parvient par lui-même, par ses statuts, par ses assemblées générales, par ses propres actions, à argumenter et à légitimer, et il ne dispose pas d'une caution institutionnelle fondatrice comparable à celle que confère une élection au suffrage universel. Son aptitude à parler au nom des habitants tient donc de sa capacité à construire une certaine figure de ceux-ci, colportée par le journal, étant entendu qu'il lui faut l'invoquer là où l'élu du suffrage universel peut agir au nom de l'intérêt général. Nous verrons plus loin comment, depuis trois ou quatre ans, le journal s'y prend pour faire valoir dans ses pages le point de vue des habitants du quartier. En attendant, nous relèverons que, sur la même période, et contrairement à ce qui se faisait auparavant, les comptes rendus des assemblées générales ordinaires que propose Quartier libre ne permettent pas de se faire une idée de la teneur des débats. Ainsi, le lecteur, bien que souvent interpellé pour rejoindre le comité, devra se contenter de brefs rappels très généraux, sinon anecdotiques, sur la mission et les actions de celui-ci et ne disposera pas de repères solides et argumentés sur le bilan des actions menées ni sur les orientations de travail retenues pour l'année à venir. D'une certaine manière, on peut se dire que le journal a aujourd'hui du mal à informer sur ce qui est au fondement de la légitimité institutionnelle du comité lui-même.

\section{L'interpellation du lecteur / de l'électeur : « Tous aux urnes !!! ॥ ou la participation à l'épreuve de l'abstention}

«S'abstenir de voter, c'est ne pas utiliser son droit civique mais aussi refuser son devoir civique. Aussi, nous invitons tous les électeurs du quartier à se rendre massivement aux urnes. » (Éditorial du n 139, février 2002, avant le premier tour de la présidentielle) Le comité peut mêler posture normative et injonction s'il s'agit de défendre des principes qui sont au fondement du pacte républicain. Cet énoncé nous permet, pour reprendre une formule de Denis Barbet, $d^{\prime}$ '«accéder aux représentations du vote» qui sont celles, ici, de militants investis dans la cause de la démocratie participative et pour lesquels l'abstention semble relever du "pôle "négatif" ( (alors que le vote 
relèverait du «normal», du «bien », du «haut») (Barbet, 2007, p. 64). Depuis une dizaine d'années, en se faisant régulièrement le support d'un appel au civisme électoral, le journal s'est trouvé un autre sujet d'interpellation, à côté de l'habitant d'une part, de l'institution municipale d'autre part: l'électeur.

La thématique apparait progressivement dans les pages de la publication à partir des municipales de 1995. Évoquées sous la forme d'une simple annonce au début, les élections font l'objet d'un véritable appel aux urnes dans le numéro de mars 1998 ( ${ }^{\circ}{ }^{113}$ ), à l'occasion des cantonales et régionales, à travers un article d'une quinzaine de lignes en bas de l'avant-dernière page: «Le 15 mars prochain, tous aux urnes !!! » Le même slogan - on notera la modalité injonctive fortement soulignée par la ponctuation - est repris dans le numéro de juin 1999 ( n $\left.^{\circ} 122\right)$ pour annoncer et présenter, sous un format comparable, les élections européennes. Il faut attendre les municipales de 2001 pour voir un tel appel figurer en une du journal. En la circonstance, le comité de quartier prend plus sérieusement en main la question en présentant, sur une demi-page, les enjeux de ces élections.

Ce type d'appel fera l'objet d'un éditorial, signé du conseil d'administration, en fin d'année 2003 ( $n^{\circ}$ 150) à propos des différentes échéances électorales de l'année suivante (cantonales et régionales, puis européennes). À l'automne 2004 puis 2005 , le journal mentionne en une, parmi d'autres "dates à retenir», celle relative aux élections des représentants de parents d'élèves dans les établissements scolaires. L'observation permet d'apprécier une autre déclinaison de la fonction de médiation du journal.

L'interpellation du lecteur n'a donc pas pour objectif, ici, de déclencher un retour vers le comité de quartier lui-même. En prenant pour cible non plus l'habitant mais l'électeur, le journal de quartier pourra, à l'occasion, devenir support d'instruction civique et adopter un registre quelque peu didactique. Ainsi, lors de la présidentielle de 2002 , non seulement seront expliquées les modalités du vote mais aussi, de manière assez précise, le rôle du chef de l'État ( $n^{\circ} 139$, février 2002). C'est le rapport aux institutions et à la nation qui fait ici l'objet de l'énoncé du journal. Le numéro de mai 2002 proposera, en première page, une petite photo du président élu accompagnée de cette légende: «Le 5 mai, le candidat démocrate a gagné », et en pages intérieures une analyse des résultats locaux des deux tours de la présidentielle. Si on sent beaucoup de prudence dans la conception de l'article et dans les commentaires, la position du comité concernant les résultats sur le secteur n'en est pas moins explicitement formulée: «On peut donc se réjouir que l'extrême droite n'ait pas fait de percée dans tous les quartiers dits populaires. Sur le quartier, cette réaction est certainement due en partie à la richesse inter culturelle. » ( $n^{\circ} 141$, mai 2002) Autre signe de sa volonté de se faire bien identifier dans l'espace public local au moment où la situation politique est critique dans le pays, le comité de quartier tiendra à assumer pleinement sa position 
dans le numéro suivant, à la suite des réactions suscitées par la photo du candidat victorieux: «Nous nous réjouissons en effet du sursaut républicain pour faire barrage à l'extrême droite. Loin de nous la pensée de faire de la publicité pour le candidat RPR. Nous estimons avoir eu une réaction tout à fait citoyenne en présentant le nouveau chef de l'État.» ( $n^{\circ} 142$, juin 2002)

La récurrence de tels appels aux urnes dans les pages du journal ne tient pas à une soudaine redéfinition des missions que se donne le comité mais au contexte abstentionniste. L'indice d'une vigilance à ce propos apparait clairement dans l'éditorial du numéro qui suit les élections européennes de 1999 quand le journal, pour la première fois d'après nos observations, fait état de cette question et l'articule à la principale raison d'être du comité: «On ne saurait évoquer la nécessité du renouveau de l'engagement sans le relier au contexte d'un absentéisme grandissant qui marque les élections les unes après les autres» ( ${ }^{\circ} 123$, juillet 1999). Un second indice du même ordre se repère dans le texte de une déjà évoqué et appelant au vote lors des municipales de 2001: "Depuis quelque temps, lors des scrutins électoraux, une nouvelle forme d'expression est apparue comme une réalité incontournable. Il s'agit de l'abstentionnisme, et le dernier référendum [sur le quinquennat] en est une parfaite illustration $»\left(n^{\circ} 133\right.$, décembre 2000$)$.

En se montrant de plus en plus vigilant sur cette question, quitte à entamer le procès des responsables politiques ${ }^{6}$, le journal témoigne de ce qu'il ne se contente plus de soutenir ni d'expliciter un principe civique : il s'est mobilisé contre ce qui lui semble être un problème de fond et parait en prise avec une problématique qui, travaillant la société française dans son ensemble, affecte tout particulièrement le quartier et la ville?. Devant cette entreprise d'information, de mobilisation, de médiation au niveau de l'infralocal, il nous semble possible de dire que le comité de quartier, via son journal et parmi d'autres acteurs associatifs, s'efforce de remédier à une défaillance de la "puissance formante» (pour reprendre une formule de Louis Quéré) de l'espace public. Cette défaillance fait que l'acte de voter, fondement de la citoyenneté républicaine, ne va pas de soi pour tout un chacun, ne prend pas le même sens pour tous. Dit autrement, en endossant une autre formule du même auteur, «l'espace public » cesse de «fabrique[r] des comportements», ici dans le domaine

6. «Cette désertion électorale est un vrai désaveu des politiques en général et du maire sortant en particulier [...]. Nos hommes politiques seraient-ils donc si peu proches des [habitants de la ville] pour que ces derniers leur refusent en bloc leur confiance? C'est une hypothèse qu'il ne faut pas écarter et nous souhaitons bonne chance au nouveau conseil municipal. » ( $n^{\circ} 134$, avril 2001)

7. Après un sursaut de la participation électorale lors de la présidentielle de 2007 , battu en brèche toutefois dès les législatives qui ont suivi, la ville et le quartier en question ont renoué avec un taux d'abstention élevé lors des élections municipales de mars 2008. Celui-ci a avoisiné $60 \%$ pour la première et dépassé ce pourcentage pour le second (sources: site Internet de la ville et Nord-Éclair des 23 avril, 7 mai, 11 et 18 juin 2007). 
précis d'une élection, évènement pourtant censé actualiser, structurer, rendre manifeste la démocratie représentative (Quéré, 1995). À l'aune de cette action locale, la participation ne s'entend plus comme un contre-pouvoir de la représentation, mais comme la caution de celle-ci. C'est là d'ailleurs un autre trait révélateur de l'évolution conceptuelle de la notion et de l'institutionnalisation de la pratique à laquelle elle renvoie (Blondiaux, 2004).

Cela dit, au cours de l'année 2007, le journal semble s'être mis en retrait d'une telle mission. Le contraste est saisissant entre les gros titres à la une des numéros 139 et $142^{8}$ («Tous aux urnes!!! »... « Les 9 et 16 juin, votons!!!») et la mention «Élection présidentielle: le 22 avril puis le 6 mai de 8 heures à 19 heures», simple "date à ne pas oublier» dans le $n^{\circ} 1719$. Et dans un éditorial qui recense différentes manifestations et animations ponctuant le printemps, les élections législatives sont assimilées à d'autres menus évènements du mois: "JUIN avec le marché et la fête des mères, les élections législatives pour élire nos députés, le $26^{\mathrm{e}}$ marathon de marche épeulois, la fête de la musique [...] » ( $n^{\circ} 172$, juin 2007). Pas un mot de plus au cours de ce premier semestre, sinon de manière très évasive, dans les éditoriaux des deux premiers numéros de l'année où le président du comité rappelle que «voter est autant un devoir qu'un droit ». La référence aux municipales de 2008 reste tout aussi allusive. Ce retrait, non explicité dans les pages de la publication, semble assez conforme à une posture de désengagement vis-à-vis de préoccupations d'ordre social et politique, auparavant structurantes dans l'action de l'association et le discours du journal.

\section{La parole aux/des habitants : l'expression populaire entre instrumentalisation, dénonciation et fragmentation}

Longtemps, Quartier libre a été un support utilisé par différentes structures pour faire passer des communiqués, des informations relatives à des services, ou pour faire connaitre publiquement des revendications (nous parlons au passé, cette fonction étant aujourd'hui moins visible) : il a relayé la parole émanant du centre social, des associations de défense de locataires, d'associations de parents d'élèves, voire, de manière très exceptionnelle, celle de salariés en grève ou d'employés de la mairie. En même temps, pendant la période faste de cet usage du journal (les années quatre-vingt-dix), il n'a offert ni courrier des lecteurs ni, sinon très exceptionnellement, tribune libre où se seraient exprimés des habitants.

Il faut attendre mai 2003 pour que le journal affiche une nouvelle vocation à côté de celle d'informer, de sensibiliser, de mobiliser: devenir un espace où

8. Respectivement, février et juin 2002.

9. Avril 2007. 
les habitants du quartier prennent la parole, en leur ouvrant ses colonnes ou en déclarant se faire leur écho. Cette possibilité est indiquée dans un court encadré au sein de la rubrique «Place publique», en dernière page du journal: "Cette rubrique concerne tous ceux qui désirent s'exprimer ou communiquer». Mais ici, le journal glisse parfois vers une restitution du discours des habitants qui amène l'analyste à s'interroger sur le sens et la portée qu'elle peut prendre dans l'espace public local.

Dans un numéro dont l'éditorial est une «lettre au maire » cinglante, signée du conseil d'administration, l'humeur des correspondants (sept habitants, photos à l'appui) tourne à l'acrimonie à propos de l'état du quartier :

Voilà 30 ans que j'habite ici et pourtant je n'en peux plus. Je m'en vais, je suis presque parti. Adieu mon quartier bien aimé. ( $n^{\circ} 147$, mai-juin 2003)

Je ne veux pas rester ici [...] mais surtout parce que dans cet immeuble, ma vie est en danger. La sécurité est une catastrophe, un commissariat de police est plus que nécessaire. J'aime la rue de [...], mais je pense quitter le quartier. (Ibid.)

Le quartier est moins plaisant qu'avant, il y a trop de magasins identiques, après le marché, les rues sont sales et remplies de détritus. (Ibid.)

Il y a des gens qui se foutent vraiment de notre gueule, ils vont le comprendre aux prochaines élections. (Ibid.)

La portion [entre deux rues] est pourrie, déguelasse [sic]. (Ibid.)

De ces extraits, on retiendra tout particulièrement la formulation et la mise en forme d'une exaspération générale. La «parole habitante» (dont la trivialité entend garantir la véracité, le «parlervrai » du peuple étant supposé témoigner de difficultés elles aussi réelles) vient ainsi appuyer le propos tenu par le comité de quartier lui-même; elle contribue à soutenir et à légitimer une montée en tension du rapport du comité avec la municipalité (Raoul, 2006). Elle officie à charge. Mais la médiation du journal ne verse-t-elle pas en l'occurrence dans l'instrumentalisation du témoignage individuel public? L'analyste est fondé à se poser la question à la lecture de cette même rubrique dans les numéros qui suivent et qui témoignent du retour à une relation apaisée. En effet, deux mois plus tard, le lecteur pourra discerner, à côté notamment d'une assez longue lettre ouverte au maire mettant explicitement en cause sa personne, des propos plus mesurés dans les «morceaux choisis» qui lui sont offerts : «Le quartier est moyen. J'aimerais qu'il y ait plus de loisirs dans le quartier»; «ll faudrait plus de policiers, des patrouilles la nuit, même de la police municipale. Maintenant, ça va mieux, c'est calme, les jeunes vont bien. Tout me plaît dans le quartier» ( $n^{\circ} 148$, juillet-août 2003). Dans le numéro suivant - entre deux articles d'une autre teneur -, la «parole habitante» est plus élogieuse encore: 
Cela fait 46 ans que j'habite le quartier et je l'aime. Les habitants, de toutes origines, sont très gentils, ils sont très corrects. [...] Je trouve qu'ily a moins de "casses" qu'avant, cela se calme, il y a du mieux. (nº 149, octobre 2003)

Je suis un lycéen de 17 ans. Je trouve que ça va dans le quartier. Il est en nette amélioration grâce à la Ville qui a créé un stade de loisirs pour qu'on puisse s'occuper. (lbid.)

J'ai remarqué que le quartier est en évolution : la propreté, le carrefour, les trottoirs; il a évolué à travers le projet urbain que je trouve satisfaisant. J'aime le côté vivant, la réalité des gens. (Ibid.)

Avant de laisser place à la rubrique qui lui succédera, «Place publique» peut rendre compte, entre autres, de réactions d'habitants vis-à-vis de problèmes liés au stationnement, aux animaux; elle fait état de "coups de gueule» à propos de risques pour la sécurité des piétons ou d'actes de vandalisme attribués aux jeunes du quartier. Si l'intention contestataire à l'encontre de la municipalité, qui a semblé animer un temps la rubrique, s'est dissipée, une mise en cause ne s'en repère pas moins à l'occasion, mais de manière plus vague:

L'ancien site LOCATRA [suit le nom de la rue] nous cause bien des tracas [...]. Faut-il attendre que le quartier saute pour réagir? ( $n^{\circ} 151$, février-mars 2004)

Il serait plus qu'urgent de trouver une solution à ce problème. En plus, la rue est sale, elle devient un dépôt d'ordures sauvages. Alors je m'adresse aux personnes qui ont pris cette décision: avant d'agir, il fallait d'abord consulter les personnes concernées. ( $n^{\circ} 152$, avril-mai 2004)

On frôle parfois la dénonciation entre voisins:

Je suis handicapée physique, on a fait un marquage au sol de stationnement handicapé dans la rue devant chez moi. Hélas, je peux rarement m'en servir. Les visiteurs des maisons avoisinantes occupent continuellement l'emplacement. ( $n^{\circ} 152$, avril-mai 2004)

Depuis le mois de janvier, une personne de la rue, mal intentionnée, met des pièges dans sa cour pour (je pense) faire fuir les chats. Un de mes chats a été blessé une fois. (Ibid.)

Le lancement dans le $n^{\circ} 154^{10}$ de la rubrique «"Tchos potins", la rubrique des Tartines» (titre qui fait résonner le parler populaire des courées du Nord), confirme la bascule de cet usage de la parole locale vers un type de registre discursif plus narratif et anecdotique, mais aussi plus insidieux lorsque sont évoquées les relations entre voisins. Cette rubrique, dans le prolongement de la précédente et sous la forme (qui se veut humoristique et allusive) de paroles recueillies auprès des habitants par trois «commères» attitrées du journal (elles en feront «toute une tartine»), évoque des problèmes du quar-

10. Septembre-octobre 2004 . 
tier (le «vivre ensemble », les conditions de vie de certains habitants, les tracasseries au quotidien...). Elle constitue également un moyen de poser des questions - sans forcément avoir un objectif autre que celui de les poser-, voire de faire des suggestions. Elle peut conduire à la mise en cause publique, l'adresse étant précisée, d'habitants au comportement inconvenant sinon agressif (vols, insultes, ébriété, drogue...) envers leurs voisins:

Mesdames et messieurs du [...] : c'est votre droit de ne pas vouloir de publicité, Des habitants de la cour [...] en ont assez des locataires de deux des maisons de la cour: ils agressent verbalement, insultent, volent le linge [...], sont régulièrement en état d'ébriété et drogués. ( ${ }^{\circ}$ 158, mars-avril 2005)

$\mathrm{Au}$ [...], le locataire du $2^{\mathrm{e}}$ étage, lorsqu'il rentre le soir, urine dans l'ascenseur. ( ${ }^{\circ} 160$, juillet-août 2005)

Un commerçant de la rue de [...] gêne beaucoup de personnes. Depuis plusieurs mois, il a ennuyé les riverains de la rue [...] avec ses camions plein d'ordures [photos des deux camionnettes à l’appui]. ( ${ }^{\circ} 170$, février 2007)

L'inconvenance peut prendre une tournure malicieuse sous un intitulé aguicheur («Porno à volonté») pour déplorer le comportement de locataires qui "ne se gênent pas de se balader nus devant leur fenêtre, voir [sic] pour ouvrir la porte d'entrée. Que dire! » Au numéro suivant, le lecteur apprendra que «les locataires ont lu [le] journal » et que «le porno a été remplacé par des rideaux et des tentures». En contribuant à refréner les ardeurs exhibitionnistes, le journal de quartier fait œuvre de médiation très pragmatique, circonstancielle.

Par une telle rubrique, le journal entend semble-t-il se montrer ouvert à l'expression des sociabilités ordinaires qui font le quotidien d'un quartier et donc, pourrait-on dire, «élargir l'espace public local »"11. Pour autant, ce genre de rubrique relève, du fait de la position énonciative adoptée, d'une forme de fétichisation (il s'agit d'aller encore plus près du vécu des habitants, du "peuple») et parfois d'instrumentalisation du témoignage individuel, à moins que ce ne soit d'une procédure d'interpellation et/ou de dénonciation publiques. En même temps, elle renforce une logique d'atomisation, de fragmentation de la parole publique et d'individualisation de la vie du quartier lors de sa mise en visibilité dans le journal. Les énoncés sont juxtaposés, mais on ne constate aucune intention de mise en débat public, de mise en confrontation de points de vue ou d'élaboration d'une parole collective. Aucune préoccupation de construire une légitimité n'est repérable. Chaque énoncé de la rubrique, voire chaque parole retranscrite ou rapportée semble avoir sa légitimité publique en soi, du seul fait qu'elle émane d'un habitant ou d'une habitante (ou prétendus tels). Le journal peut donc, tout en s'instaurant en

11. En écho à une problématique traitée notamment dans l'ouvrage collectif coordonné par Bastien François et Érik Neveu (1999). Voir la première partie de l'ouvrage. 
«puissance de cautionnement» (Boltanski, 1984, p. 15), instituer en problème public (local) les implications de certaines pratiques individuelles considérées par les responsables de la publication comme dérogeant à la normalité.

Considérant le discours d'un journal de quartier en tant qu'il contribue à donner forme et sens à un espace public local, nous nous sommes ici attaché à étudier l'évolution de sa fonction de médiation, en particulier au cours de la dernière décennie. Quelques enseignements majeurs, corrélés entre eux, nous paraissent ressortir de cette investigation et nous permettent de mettre en évidence une forme de dépolitisation de cette publication.

Le journal «donne la parole» aux habitants ou entend s'en faire directement l'écho. En retour, il semble que la "parole habitante» confère un gage d'authenticité et de sincérité au discours du média, en même temps qu'elle vient conforter sa légitimité et donc celle du comité de quartier à faire état des situations ordinaires des habitants, à parler en leur nom, à les représenter. Mais le journal s'en tient à une forme de restitution atomisée de cette parole, il ne cherche pas à la construire en parole collective et à l'inscrire dans une perspective de débat. Résonne ici l'observation faite par Dominique Cardon et Jean-Philippe Heurtin au cours d'une recherche portant sur une émission de radio publique: «L'authenticité du témoignage lui confère une autorité qui le soustrait à la discussion argumentée.» (Cardon, Heurtin, 1999, p. 89)

Mais c'est de manière plus générale que la production discursive du journal tend aujourd'hui à faire prévaloir une vision factuelle, événementielle et, par là, fragmentée de l'espace public local. Non content de renoncer à la mission d'information et de sensibilisation civique qu'il a assumée quelques années, le journal affiche aujourd'hui une orientation polémique et un ton moraliste bien plus qu'il ne fait montre d'un souci d'investigation et d'explication, d'une force d'analyse et d'argumentation. En d'autres termes, il ne parvient plus à «monter en généralité» à propos des différentes problématiques du quartier, à inscrire les phénomènes qu'il évoque dans un cadre de catégorisation et d'interprétation permettant de "gagner en intelligibilité» (François, Neveu, 1999, p. 32). Bref, c'est la capacité du journal à instruire le débat public en offrant au lecteur un regard structurant et politique qui s'est altérée, c'est sa fonction de médiation critique qui tend à se déliter. Pour conclure, nous dirons qu'en révélant une telle tendance, l'analyse du texte du journal invite à une investigation d'un autre ordre, notamment dans une perspective comparative. 


\section{Références}

Authier Jean-Yves, BACquÉ Marie-Hélène, GuÉRIN-PACE France éd., 2007, Le quartier. Enjeux scientifiques, actions politiques et pratiques sociales, Paris, La Découverte.

BARBET Denis, 2007, "Quand les mots de l'abstention parlent des maux de la démocratie», Mots. Les langages du politique, $\mathrm{n}^{\circ} 83$, Dire la démocratie aujourd'hui, p. 53-67.

BLONDIAUX Loïc, 2000, «La démocratie par le bas. Prise de parole et délibération dans les conseils de quartier du vingtième arrondissement de Paris ", Hermès, $n^{\circ}$ 26-27, p. 323-338.

- 2005, «Démocratie délibérative et démocratie participative. Une lecture critique», Démocratie participative et gestion de proximité, M.-H. Bacqué, Y. Sintomer éd., Paris, La Découverte.

BoltANSKI Luc, 1984, "La dénonciation", Actes de la recherche en sciences sociales, $\mathrm{n}^{\circ} 51$, p. 3-40.

BonnAfous Simone, 2006, "L'analyse du discours», Sciences de l'information et de la communication. Objets, savoirs, discipline, S. Olivesi éd., Grenoble, Presses universitaires de Grenoble, p. 213-228.

Boure Robert, LefebVRe Alain, 2000, «Télévisions “locales” et territoires en mouvement. Vers un programme de recherche », Hermès, n 26-27, p. 265-282.

CARdon Dominique, Heurtin Jean-Philippe, 1999, "La critique en régime d'impuissance. Une lecture des indignations des auditeurs de France-Inter», Espaces publics mosaïques, B. François, É. Neveu éd., Rennes, Presses universitaires de Rennes, p. 85-119.

Delforce Bernard, Noyer Jacques, 1999, «Pour une approche interdisciplinaire des phénomènes de médiatisation. Constructivisme et discursivité sociale», Études de communication, $\mathrm{n}^{\circ} 22$, p. 13-39.

FrançoIs Bastien, NeVEu Érik éd., 1999, Espaces publics mosaïques, Rennes, Presses universitaires de Rennes (Introduction: «Pour une sociologie politique des espaces publics contemporains », p. 13-58).

INSEE, 2007, Les Dossiers de Profils, $\mathrm{n}^{\circ} 88$, Regards sur les quartiers en Nord-Pas-deCalais, [en ligne] [ULR: http://www.insee.fr/fr/insee_regions/nord-pas-de-calais/ themes/dossiers_de_profils/DP88.pdf], consulté le 17 mars 2009.

KRIEg-Planque Alice, 2006, " “Formules” et “lieux discursifs” : propositions pour l'analyse du discours politique», Semen, $n^{\circ} 21$, p. 19-47.

Lefebvre Rémi, Nonjon Magali, 2003, "La démocratie locale en France. Ressorts et usages », Sciences de la société, $\mathrm{n}^{\circ}$ 60, p. 9-28.

MaINGUENeAu Dominique, 2005, "L'analyse du discours et ses frontières », Marges linguistiques, $\mathrm{n}^{\circ} 9$, p. 64-75.

NeVEU Catherine, 2003, Citoyenneté et espace public. Habitants, jeunes et citoyens dans une ville du Nord, Villeneuve d'Ascq, Presses universitaires du Septentrion. 
BRUNO RAOUL

- 2007, "Le quartier des politistes», Le quartier. Enjeux scientifiques, actions politiques et pratiques sociales, J.-Y. Authier, M.-H. Bacqué, F. Guérin-Pacé éd., Paris, La Découverte, p. 32-40.

QUÉRÉ Louis, 1995, "L'espace public comme forme et comme événement», Prendre place. Espace public et culture dramatique, I. Joseph éd., Paris, Recherche / Plan urbain (colloque de Cerisy), p. 93-110.

RAoul Bruno, 2006, "Une voie d'expression dans la ville: un journal de quartier dans l'espace public (local). Un regard sur la "démocratie participative" à Roubaix», Sciences de la société, $\mathrm{n}^{\circ}$ 69, Démocratie participative en Europe, p. 279-290. 